\title{
THE CONVECTION ZONE AND OSCILLATIONS
}

\author{
V.A.BATURIN, I.V.MIRONOVA AND S.V.AYUKOV \\ Sternberg Astronomical Institute, \\ Universitetsky pr., 13, Moscow, Russia
}

\section{Introduction}

We study the influence of the structure and physics of the uppermost superadiabatic layers on the oscillation spectrum of $p$-modes. These modes are trapped inside the Sun by reflecting at the upper layers of the Sun, so these layers are important for the oscillation spectrum. Indeed, the main difference between the computed and observed frequencies seems to come from these layers (Christensen-Dalsgaard, 1985; Christensen-Dalsgaard and Thompson, 1997).

We discuss three sources of uncertainties: atmospheric opacity, convection description and the dynamic terms in pressure, i.e., the turbulent pressure.

\section{Atmospheric opacity}

The density profile in the solar radiative atmosphere is known with a significant uncertainty chiefly originating from the assumed opacity tables. An increase of the atmospheric opacity leads to quite substantial changes of the oscillation spectrum which can be interpreted as an effective increase of the acoustic radius of the Sun (Christensen-Dalsgaard, 1990; Baturin and Mironova, 1995). The resulting spectrum modifications may remove a significant part of the differences between the observed and computed frequencies. This approach has several limitations. Firstly, the opacity can hardly be amplified to arbitrary values. Secondly, it cannot explain the frequency differences for the modes between 2 and $3 \mathrm{mHz}$ (not to mention the high-frequency end of the spectrum). Nevertheless, opacity changes in the atmosphere provide an example of a prominent effect on the oscillation spectrum.

\section{Theory of convection}

The next potential cause for the frequency differences is the structure of the uppermost superadiabatic region of the solar convection zone. Given the fixed entropy at the bottom of the convection zone the problem is reduced to an adequate description of the structure of the superadiabatic layers. The possible influence of the convective description on the spectrum of oscillations was studied in a series of papers (e.g. Baturin and Mironova, 1995; Monteiro et al., 1995; Rosenthal, 1997). We restrict our 


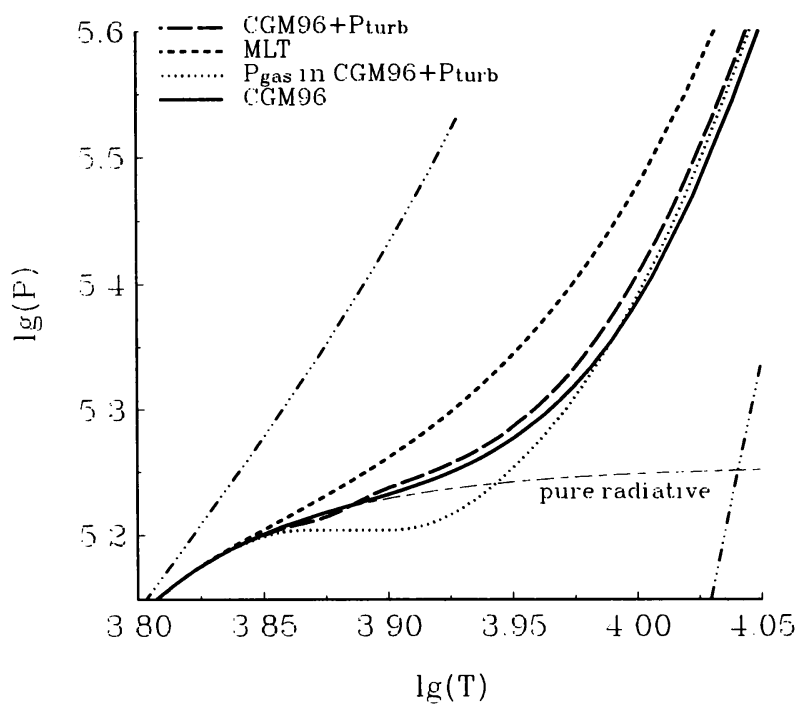

Figure 1. Different convection predictions in the temperature-pressure plane. Only superadiabatic part of the convection zone is shown. MLT-mixing length theory; CGM96-Canuto, Goldman and Mazzitelli (1996) theory. Models with turbulent pressure are plotted as well. The limiting adiabats are dot-dashed.

considerations to convection theories with horizontal averaging and local convective formulations. A convection theory should predict the temperature gradient in the superadiabatic layers. The region of superadiabatic convection is very shallow, only a couple of hundred kilometers in height. Fig. 1 illustrates the role of the convection theory in describing the transition from the top boundary of the convection zone (minimum of entropy) to the deep adiabatic layers. MLT predicts a fairly smooth transition between those two adiabats, whereas CGM96 theory (Canuto et al., 1996) corresponds to a sharper transition. It is also clear that there is not much room left for other completely different convective descriptions, and the CGM96 description is a physically reasonable example of the sharpest transition in the superadiabatic layers.

The influence on the frequency spectrum is shown in Fig. 2. Sharpening the convective transition (i.e., increasing the temperature gradient) leads to a desirable effect on the frequencies, but the amplitude is not as large as in case of the opacity correction. Another variant of the Canuto and Mazzitelli theory has been plotted in Fig. 2 to demonstrate the possibility of amplifying the effect (CMz91). However, this modification corresponds to extremely large variations of the temperature gradient which seem to be unrealistic. But it is worth noticing that changes of the description of convection are able to alter the frequency behaviour in $2-3 \mathrm{mHz}$ range.

\section{Turbulent pressure}

The most intriguing effect is the dynamic features of convection (Antia and Basu, 1997; Kosovichev, 1995; Mironova and Baturin, 1997; Nordlund and Stein, 1996). More exactly, we consider the additional pressure term in the hydrostatic equation (turbulent pressure). We do not attempt a detailed description of the exact nature 

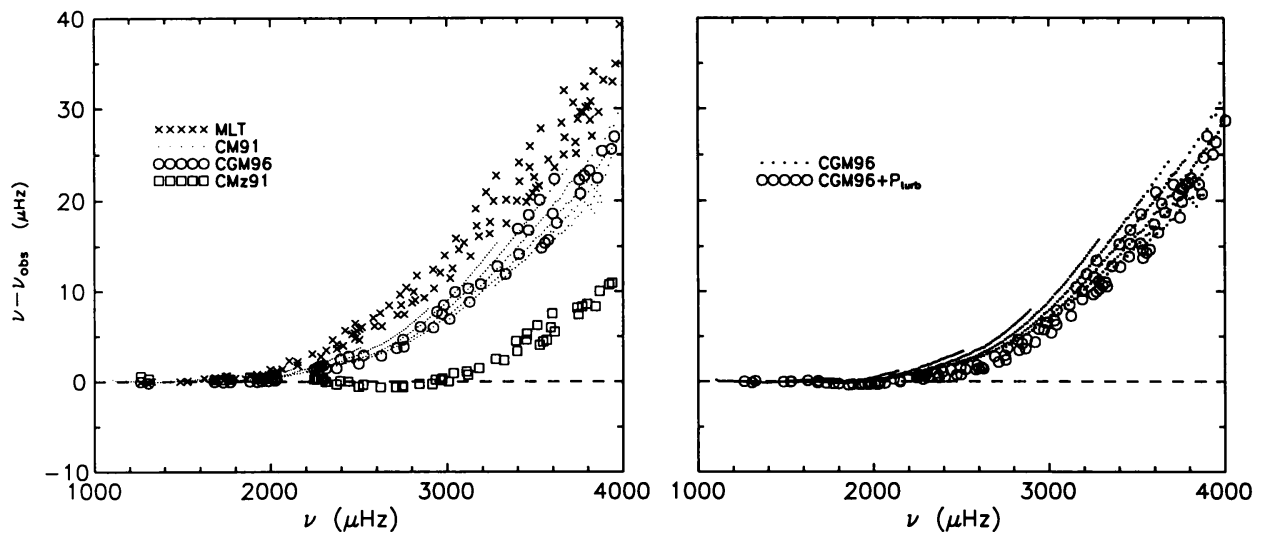

Figure 2. (left) Oscillation spectrum of models with different convection descriptions. CM91 and CMz91 - convection theory by Canuto and Mazzitelli (1991). See also caption to Fig. 1 for abbreviations.

Figure 3. (right) Effect of inclusion of turbulent pressure on model frequencies.

of this term or of the properties of turbulent convection. Instead of this we estimate the possible effect of the additional pressure term on the oscillational spectrum. To achieve this we have computed a model with a turbulent pressure term according to Canuto, Goldman and Mazzitelli (1996).

The structural changes are easily predictable. The gas component of pressure is reduced, as is density, but the total pressure is roughly the same. The effect is rather strong (turbulent pressure can contribute as much as 12 per cent to the total pressure), but strictly localized - the stratum where turbulent pressure appears is five times shallower than the superadiabatic region. This explains the rather surprising result--the frequencies of the model modified by turbulent pressure are close to the "basic" model frequencies (Fig. 3.)

This result was obtained using Canuto, Goldman and Mazzitelli formulation. We did not investigate the question whether other turbulent pressure descriptions are similarly localized.

Turbulent-pressure terms also occur in oscillation equations used for the frequency calculations. This complex effect can be simulated by changing the adiabatic compressibility $\Gamma_{1}$. We have recalculated the theoretical frequencies with the so-called reduced $\Gamma_{1}$ in the model with turbulent pressure (following a recipe by Rosenthal, 1997) and found that an influence on frequencies is quite small. This might be explained by the fact that the region where turbulent pressure has some effect is very narrow; also, the acoustic waves are predominantly evanescent in these layers.

\section{Summary}

The possibilities for improving the theoretical $p$-mode spectrum within the framework of local convective models and adiabatic oscillations are mostly exhausted by the effects considered. A combination of these effects can reproduce the observational frequencies up to $3 \mathrm{mHz}$, but fails to do so in the high-frequency range. For further improvement it may be feasible to consider non-adiabatic effects and horizontally in- 
homogeneous convection theories. The influence of the dynamic features of convection seems to be less significant than expected.

The authors thank J. Christensen-Dalsgaard for very useful comments.

\section{References}

Antia H.M., Basu S.: 1997, in F.P.Pijpers, J.Christensen-Dalsgaard, and C.S.Rosenthal (eds), Proceedings of Solar Convection and Oscillations and their Relationship, Kluwer, p. 51.

Christensen-Dalsgaard J.: 1985, in D.O.Gough (ed.), Seismology of the Sun and the Distant Stars, Reidel Publ. Co., Dordrecht, Holland, 169, p. 23.

Christensen-Dalsgaard J.: 1990, in G.Berthomieu, M.Cribier (eds), Inside the Sun, Kluwer, Dordrecht, p. 305.

Christensen-Dalsgaard J., Thompson M.J.: 1997, Monthly Notices Roy. Astron Soc. 284, 527.

Baturin V.A., Mironova I.V.: 1995, Astronomy reports 39, 107.

Canuto V.M., Goldman I., Mazzitelli I.: 1996, Astrophys.J. 473, 550.

Canuto V.M., Mazzitelli I.: 1991, Astrophys.J. 370, 295.

Kosovichev A.G.: 1995, in J.T.Hoeksema, V.Domingo, B.Fleck, B.Battrick (eds), Helioseismology: Proceedings of the 4th SOHO Workshop, 1, ESA SP-376, ESTEC, Noordwijk, p. 165 .

Mironova I.V., Baturin V.A.: 1997, in F.-X.Schmider and J.Provost (eds), Proceedings of the IAU Symp. No.181, Sounding Solar and Stellar Interiors, Nice Observatory (in press).

Monteiro M.J.P.F.G., Christensen-Dalsgaard J., Thompson M.J.: 1996, Astron. Astrophys. 307, 624 .

Nordlund A., Stein R.F.: 1996 in A. Noels et. al. (eds), Proceedings of the 32d Liege Colloquium, Liege: Univ. Liege, p.75.

Rosenthal C.S.: 1997, in F.P.Pijpers, J.Christensen-Dalsgaard, and C.S.Rosenthal (eds), Proceedings of Solar Convection and Oscillations and their Relationship, Kluwer, p.145. 